\title{
Development of germinal centres in the spleen in infants related to birth and unexpected death
}

\author{
A. J. AL BARZANJI, S. R. PENNY, AND J. L. EMERY \\ From the Department of Pathology, Children's Hospital, Sheffield
}

SYNOPSIS The development of germinal centres in the spleen has been estimated in a series of 266 children dying in hospital and 205 children presenting as unexpected death in infancy. The prevalence of germinal centres in the spleen increases rapidly after birth. The percentage of Malpighian bodies showing germinal centres in the spleen is greater in all categories of our classification of death in children dying unexpectedly at home when these are compared with hospital deaths. It is concluded that the children presenting as unexpected death in infancy show no evidence of any deficiency in cellular immunological response and may well have been subjected to increased or precocious antigenic stimulation.

In a study of the lymphatic tissues of the lung, Emery and Dinsdale (1973) observed that lymphoreticular aggregates develop only after birth and that they did so in greater quantity in children who were found dead at home ('cot death') than in other child deaths (Emery and Dinsdale, 1974). They suggested that infants presenting in the 'cot death' category could well have been brought up in a more antigenic-stimulating environment than the others. Baak and Huber (1974) studied the thymus in children in the 'cot death' category and reported that in such children the thymus shows a higher percentage volume of cortex and a lower concentration of lymphocytes in the cortex and medulla. We thought that a survey of the germinal centres in the spleen might throw some light on the cellular immunological state in such infants.

The germinal centres in the spleen are believed to be the result of an immunological response to foreign antigens, the sites of the formation of antibody-containing plasma cells. The presence of germinal centres in the spleen and other lymphatic tissue does not necessarily mean that infection was the antigenic stimulus but it has been shown by many that infection is a definite common stimulus (Jacobson and Thorbecke, 1968; Pettersen and Rose, 1968; Abe and Ito, 1973; Anderson, 1973).

We have examined postmortem tissues of the spleen in an attempt to answer the question, 'What is the pattern of development of germinal centres in

Received for publication 30 December 1975 the spleen in infants, and is there any difference between the pattern in those dying in hospital and those presenting as unexpected deaths at home?'

\section{Material and methods}

The material used was taken from the slide files of the Department of Pathology at the Sheffield Children's Hospital and was a sequential series from infants dying during the years 1969-74. Stillborn fetuses and children dying within four days of birth were excluded. Two sequential series were taken, each of approximately 200 cases, making 471 cases in all. These comprised 266 hospital deaths and 205 children who presented as unexpected death in infancy. Blocks of the spleen were taken as a routine part of the necropsy; they were taken from the centre of the organ and included the capsule. In infant spleens, the whole of the organ was transected; in older children, the block included about half of the transection. The blocks and tissues were fixed in buffered formalin, routinely processed, cut in wax at $6 \mu$, and stained with Masson's trichrome or haematoxylin and eosin.

A complete scan of each section was made, counting and scoring all Malpighian bodies, which were then divided into the following categories (see figs 1 and 2):

1 Malpighian bodies with a uniform mat of lymphocytes;

2 bodies with a fairly uniform mat of lymphocytes, but within the lymphatic mass are larger pale cells 


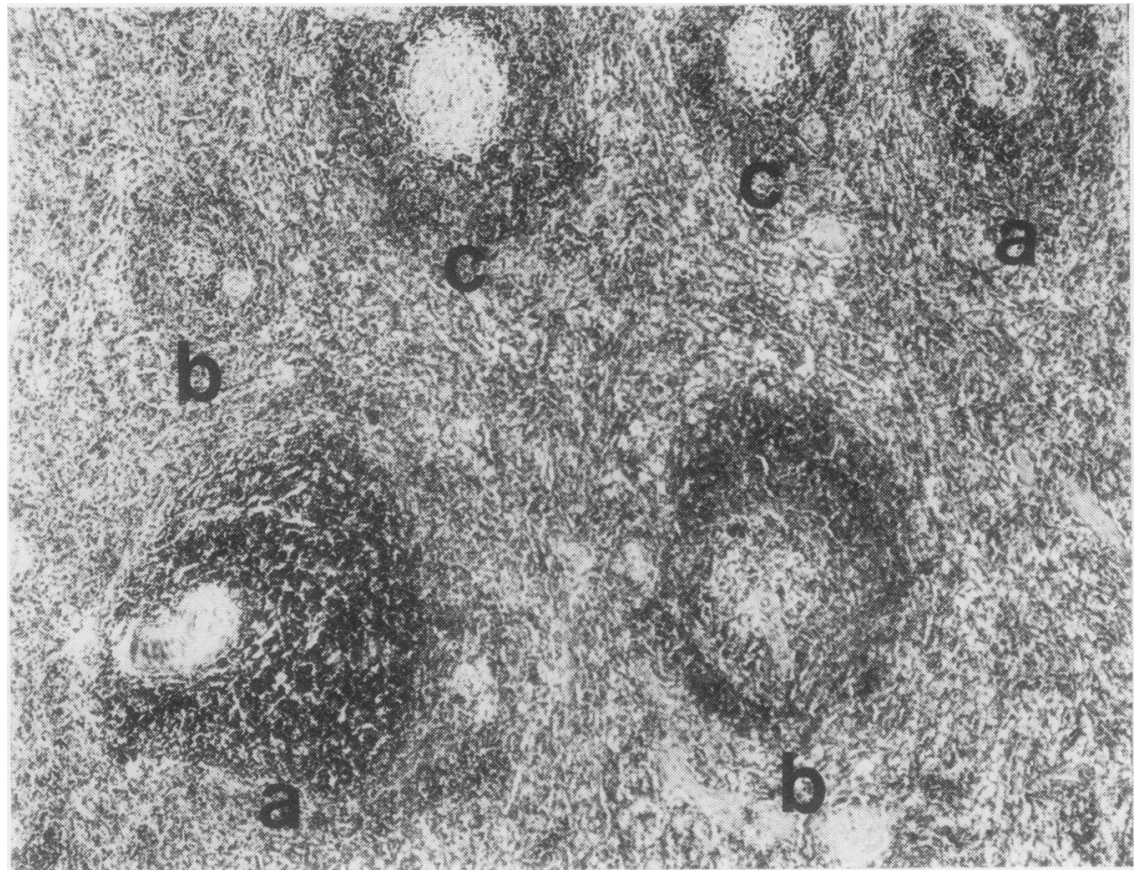

Fig 1 A section of spleen showing six Malpighian bodies (a) withno germinal centres (b) with a simple centre (G1); and (c) with large, definite centres $(G 2)$.

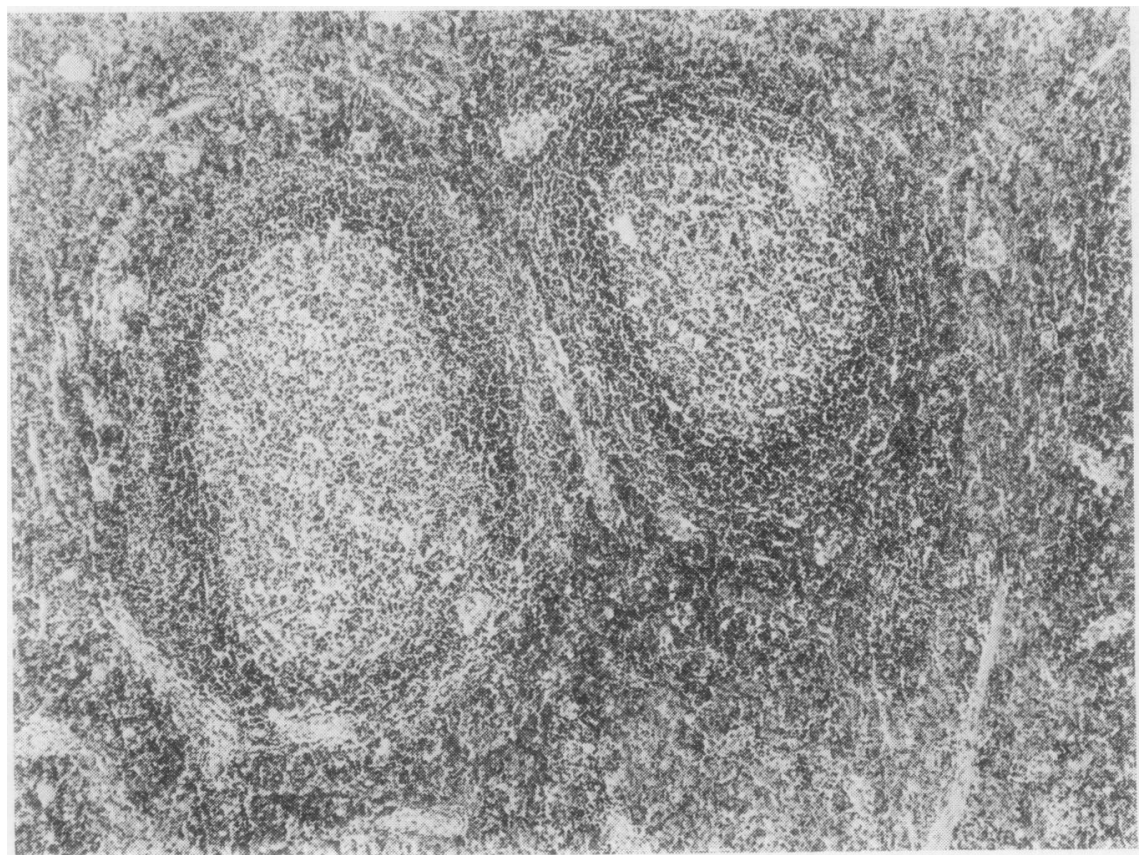

Fig 2 A section of spleen showing two Malpighian bodies containing large germinal centres of the type specified as type $\mathrm{G} 2$ in category 4.

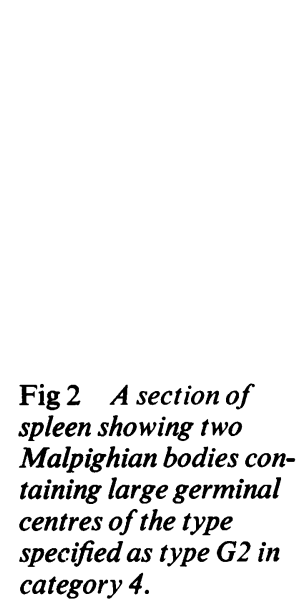

with clear cytoplasm producing a picture usually referred to as a 'starry sky';

3 Malpighian bodies in which the lymphocyte

masses contain a distinct group of large pale cells, often with nuclear and other debris (G1); 4 Malpighian bodies containing larger pale areas of 
cells within the lymphocyte masses (G2), the centres having a different structure from the surrounding lymphatic masses and with an apparently clearcut compressed edge of the surrounding cells (Millikin, 1970).

It was appreciated that the relative size of the centres as seen in random sections depends on the level of cut. Serial sections had indicated that the firm edge of the G2 type centres was generalized over the centre, and thus we felt justified in using single random sections for this study.

Slides were examined by two workers independently, neither having knowledge of the age or category of death of the child. Every Malpighian body was counted in each slide, and the type and number of each category were expressed as a percentage. Photographs and diagrams of the categories were continually available beside the working microscopes. After the differential count had been made the results were correlated with the types of death.

The deaths in this series were divided roughly into four groups:

Group A children with gross deformities in whom death was essentially inevitable and not amenable to treatment;

Group B children in whom necropsy revealed a definite severe state, these states being essentially treatable, ie, the explained, though treatable diseases;

Group $\mathrm{C}$ children with evidence of some disease, such as tracheitis, but in whom the morbid anatomical findings were, of themselves, inadequate to explain death;

Group D children in whom no significant histological disease state was found.

Of the 266 children dying in hospital, 139 were of group A and 127 of group B. Of the 205 children dying unexpectedly at home, 3 were in group $A$, 70 in group B, 90 in group C, and 42 in group D.

\section{Results}

The ages of the children studied ranged from 5 days to 90 weeks. In the unexpected death in infancy group, the small number of children dying in group A was discarded.

In the analysis of the material, reproducibility tests on the sections showed complete agreement in the assessment of germinal centres of groups 3 and 4 (G1 and G2). There was less agreement concerning the presence or absence of a 'starry sky'. Very few

100

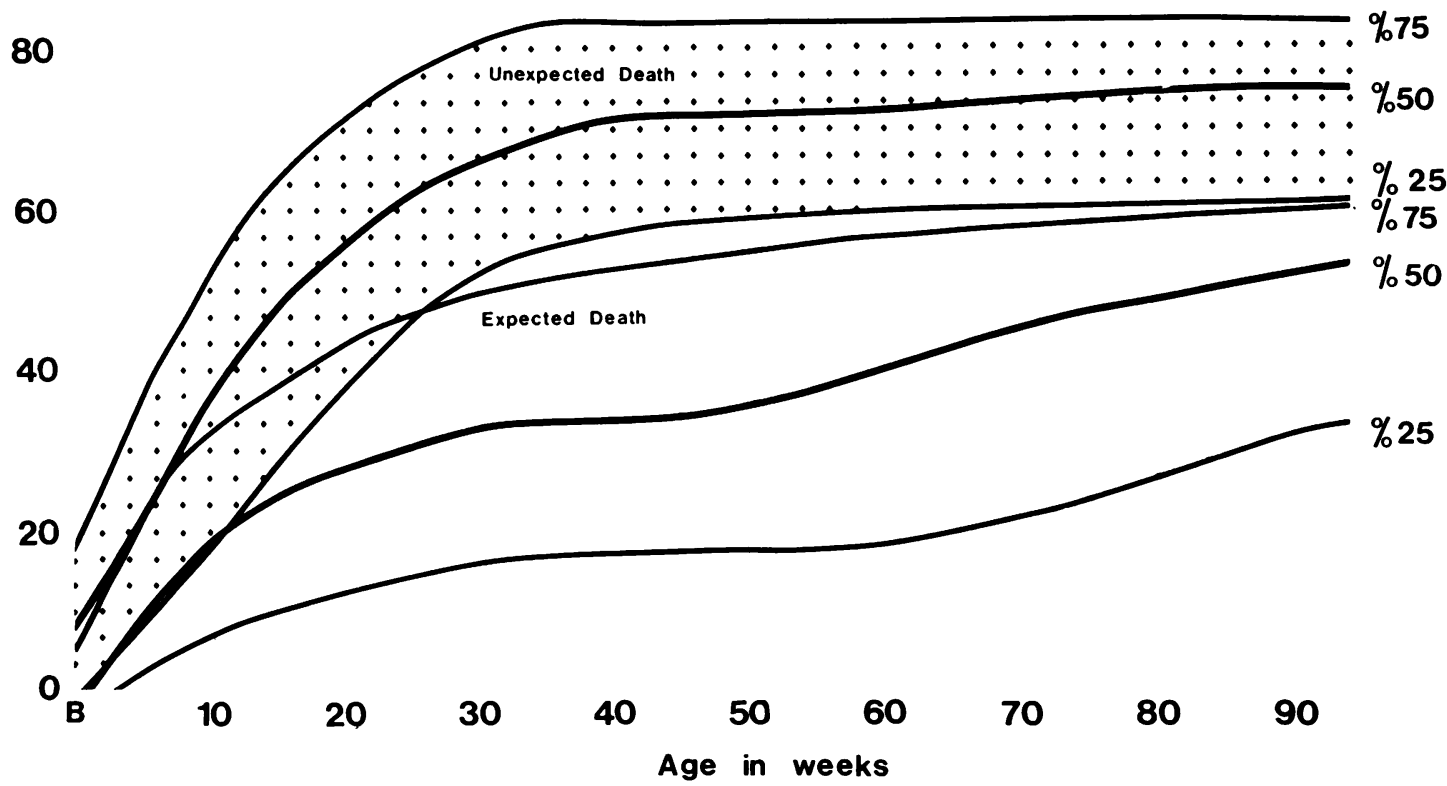

Fig 3 Superimposition of the percentage of Malpighian bodies showing germinal centres in expected and unexpected eaths. 
spleens showed the presence of a starry sky in the absence of germinal centres and these were usually in the younger children. Thus, for the purpose of the present analysis, the data on starry sky, in isolation, are not presented.

In parallel with the spleens reported here, the sections from many stillbirths and neonatal deaths were counted. All but a very few of these were devoid of germinal centres. Intrauterine infection and the gestational ages of the infants could be important factors at this stage. As it apparently takes three or four days for germinal centres to develop it seemed best to confine our observations to children dying 5 days or more after birth and to leave the perinatal period for separate study.

The findings over the whole range of children up to the age of approximately 100 weeks are shown in fig 3, where the mean line and 25th and 75th percentiles are shown. The figure shows the total germinal centres in expected deaths, combining both large and small germinal centres. The same findings are shown in unexpected deaths, and the two are superimposed. The figure shows a steep rise in the occurrence of germinal centres after birth in both groups of children but the rise is apparently both earlier and greater in the children presenting as unexpected deaths.

The early age group is examined in a different way in fig 4 where the data for large germinal centres (G2) from all children dying between 5 days and 40 weeks after birth are shown. Each symbol on the graph represents the mean point of approximately 10 cases taken in sequential age groups. This also shows that the development of the centres is a postnatal phenomenon. There appears to be a fairly rapid increase in the incidence of these large germinal centres in both the expected and unexpected deaths during the first eight weeks after birth. After this age there is a difference in incidence in expected and unexpected deaths, for from the age of 12 weeks all of the mean points are lower in the expected than in the unexpected deaths.

An attempt was made to associate the prevalence of germinal centres with the death groups A, B, C, and $D$. In specific disease states the numbers became too small for statistical significance in the individual age groups and there is considerable case variation, but, in general, the situation appears to be the same as that shown in figure 3. Both $A$ and $B$ group deaths in hospital show a lower proportion of Malpighian bodies containing germinal centres than do $A$ and $B$ deaths at home. In the unexpected deaths there is no clear difference in the incidence of germinal centres between the $\mathbf{B}, \mathbf{C}$, and $\mathbf{D}$ groups, ie, $D$ group deaths show the same increase in germinal centres as the Bs and Cs.

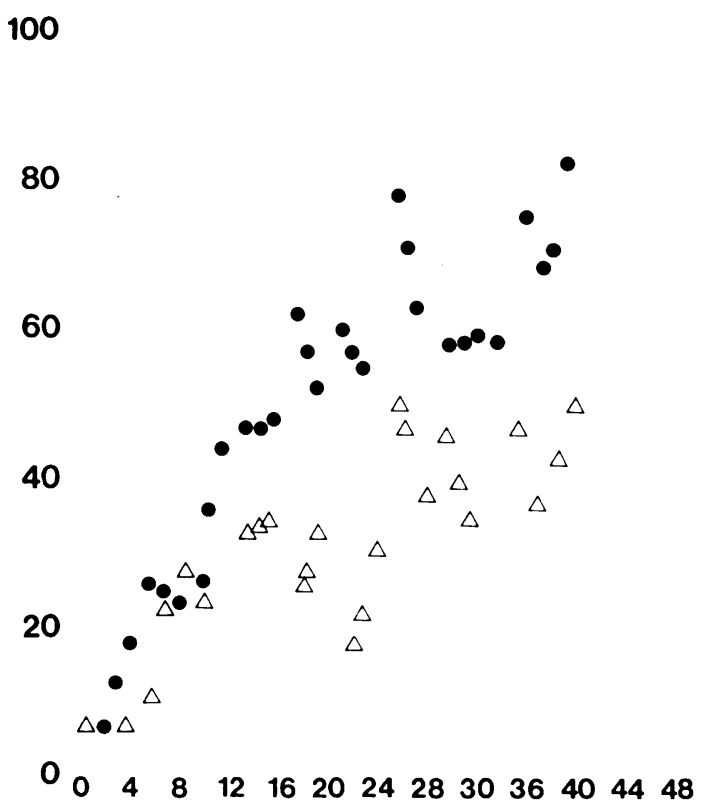

Age in Weeks

Fig 4 Percentage of Malpighian bodies showing large germinal centres of type $G 2$ in expected $(\Delta)$ and unexpected $(\bullet)$ deaths in the immediate postnatal period. Each symbol represents the mean findings of approximately 10 cases taken in an age sequence.

\section{Discussion}

There is ample evidence that germinal centres are produced in the spleen as a result of bacterial and viral infections and are a response to any repetitive antigenic stimulation. Their existence provides morphological evidence that an immune reaction has occurred. A high index of germinal centres indicates that there is significant production of new cells which are antibody-containing (Fliedner et al, 1964). There is some evidence to suggest that it takes approximately three days after antigenic stimulation before germinal centres appear in the spleen and that their incidence reaches a peak around the fifth to seventh day (Jacobson and Thorbecke, 1968; Anderson, 1973). It appears from our general figures, of both the expected and unexpected deaths, that germinal centres in the spleens of infants are almost entirely the result of postnatal antigenic stimulation.

In discussing the significance of the findings in the spleen related to unexpected death in infancy, it must be appreciated that the concept of these babies being perfectly healthy and being found unexpectedly 
dead is a popular one which has grown for a variety of reasons, but that concept is quite contrary to our experience (Emery and Cowley, 1956; Sinclair-Smith et al, 1975). In only two of the 205 unexpected death cases in this series (all of which were referred to the Coroner) did our social worker fail to show any history of symptomatology indicating infection and evidence of disease. There were many different diseases active in children of the $\mathbf{A}$ and $\mathbf{B}$ groups, but in the other groups, death, although not adequately explained, must not be looked upon as being due to a single cause. The evidence of an apparently greater immunological response, as shown in our present study by an increase in germinal centres in children found unexpectedly dead, may be related more to general environmental factors in these children than to any terminal cause of death. Our findings in the spleen parallel those in the lung, where an increased incidence of lymphoreticular foci in the unexpected death group was found. We thus have two indicators that the children who fall into the category of unexpected death at home have either responded more actively to normal environmental antigenic stimulation or have been submitted to more such stimuli than the children who died in hospital. The latter would appear to be the more likely from the work of Jericho et al (1971), who showed that in newborn pigs the amount of lymphoreticular-cell reaction in the lung is directly related to the amount and concentration of the environmental antigenic stimuli.

Among the many theories of the cause of unexpected death in infancy, that of immunological inadequacy has frequently been advanced. Estimation of the immunoglobulin levels in the blood of such infants shows variable results (Valdes-Dapena, 1973) but certainly no evidence of a lack of immunoglobulins. Our findings in the spleen, as in the lung, are more in accord with an enhanced immunological state and lend support to a concept of these children having a precocious development of allergic reactions or hypersensitivities rather than the reverse.

We wish to acknowledge the technical assistance provided by the Foundation for the Study of Infant Deaths, and the secondment of one of us (AJB) from the University of Baghdad.

\section{References}

Abe, K. and Ito, T. (1973). Fine structure of germinal centrcs of the splenic lymphatic tissue of the mouse, with special reference to the occurrence of peculiar intercellular globules in the light zone. Virchows Arch., Abt. B, 12, 259-272.

Anderson, J. C. (1973). The induction of germinal centres in germ-free chickens. J. Path. Bact., 109, 251-257.

Baak, J. P. A. and Huber, J. (1974). Morphometry of the thymus in S.I.D.S. In S.I.D.S., 1974. Proceedings of the Francis E. Camps International Symposium on Sudden and Unexpected Deaths in Infancy, edited by R. R. Robinson, pp. 27-41. Canadian Foundation for the Study of Infant Deaths, Toronto.

Emery, J. L. and Cowley, E. M. (1956). Clinical histories of infants reported to the Coroner as cases of sudden unexpected death. Brit. med.J., 2, 1518-1521.

Emery, J. L. and Dinsdale, F. (1973). The postnatal development of lymphoreticular aggregates and lymph nodes in infants' lungs. J. clin. Path., 26, 539-545.

Emery, J. L. and Dinsdale, F. (1974). Increased incidence of lymphoreticular aggregates in lungs of children found unexpectedly dead. Arch. Dis. Childh., 49, 107-111.

Fliedner, T. M., Kesse, M., Cronkite, E. P., and Robertson, J. D. (1964). Cell proliferation in germinal centers of the rat spleen. Ann. N.Y. Acad. Sci., 113, 578-594.

Jacobson, E. B. and Thorbecke, G. J. (1968). Relationship of germinal centers in lymphoid tissue to immunologic memory. Lab. Invest., 19, 635-642.

Jericho, K. W. F., Austwick, P. K. C., Hodges, R. T., and Dixon, J. B. (1971). Intrapulmonary lymphoid tissue of pigs exposed to aerosols of particles of Salmonella oranienburg, of Mycoplasma granularum, and to an oral inoculum of larvae of Metastrongylus apri. J. comp. Path., 81, 13-21.

Millikin, P. D. (1970). Epithelioid germinal centres in the human spleen. Arch. Path., 89, 314-320.

Pettersen, J. C. and Rose, R. J. (1968). Marginal zone and germinal center development in the spleens of neonatally thymectomized and non-thymectomized young rats. Amer. J. Anat. (Lond.), 123, 489-500.

Sinclair-Smith, C. C., Dinsdale, F., and Emery, J. L. (1975). Confluent lymphoreticular aggregates in the bronchi of children-'mural bronchitis'. Brit. J. Dis. Chest, 69, 279-286.

Valdes-Dapena, M. A. (1973). Sudden, unexpected and unexplained death in infancy-a status report. New Engl. J. Med., 289, 1195-1197. 